\title{
Nonlocal nonlinear phononics
}

\author{
M. Henstridge', M. Först', E. Rowe', M. Fechner ${ }^{1}{ }^{1}$ and A. Cavalleri ${ }^{1,2}{ }^{\circledR}$
}

\begin{abstract}
Nonlinear phononics relies on the resonant optical excitation of infrared-active lattice vibrations to induce targeted structural deformations in solids. This form of dynamical crystal structure design has been applied to control the functional properties of many complex solids, including magnetic materials, superconductors and ferroelectrics. However, phononics has so far been restricted to protocols in which structural deformations occur within the optically excited volume, sometimes resulting in unwanted heating. Here, we extend nonlinear phononics to propagating polaritons, spatially separating the functional response from the optical drive. We use mid-infrared optical pulses to resonantly drive a phonon at the surface of ferroelectric LiNbO ${ }_{3}$. Time-resolved stimulated Raman scattering reveals that the ferroelectric polarization is reduced over the entire $50 \mu m$ depth of the sample, far beyond the micrometre depth of the evanescent phonon field. We attribute this effect to the anharmonic coupling between the driven mode and a polariton that propagates into the material. For high excitation amplitudes, we reach a regime in which the ferroelectric polarization is reversed, as revealed by a sign change in the Raman tensor coefficients of all the polar modes.
\end{abstract}

$\square$ enerally, optical radiation tuned to frequencies immediately above that of a transverse optical phonon, where the real part of the dielectric function $\varepsilon_{1}(\omega)<0$, is evanescently screened and does not propagate. The interaction of the light field with the material, and the ability to manipulate its properties ${ }^{1-10}$, remains inherently local. This is generally true in centrosymmetric materials, in which not only the driven vibration but also Raman-active modes that are displaced by anharmonic mode couplings do not propagate. However, if the material breaks inversion symmetry, the optically excited mode can exert a displacive force also onto symmetry-odd modes, which can propagate as polaritons. This type of phonon-polariton coupling opens up a broad class of phenomena that we term nonlocal nonlinear phononics.

Polariton excitation through phononics builds on extensive previous work in which polaritons were excited using impulsive stimulated Raman scattering from propagating near-infrared pulses ${ }^{11,12}$. In these studies, steering, focusing and amplification of terahertz radiation was shown to occur in regions of the crystal beyond those traversed by the excitation pulse ${ }^{13,14}$. However, even in cases in which phase matching was optimized, polariton fields never reached amplitudes that could alter material functional properties. In this Article, we show that nonlocal nonlinear phononics can alter the ferroelectric polarization, and even reach a regime in which the polarization is reversed ${ }^{15}$.

In the experiments reported here, $\mathrm{LiNbO}_{3}$ single crystals were illuminated with mid-infrared pulses of $20 \mathrm{THz}$ central frequency and $150 \mathrm{fs}$ duration, with fluences that extended up to nearly $200 \mathrm{~mJ} \mathrm{~cm}^{-2}$, that is, peak electric fields up to $30 \mathrm{MV} \mathrm{cm}^{-1}$. Previous work has shown that under these conditions a sizable reduction of the ferroelectric polarization, and even a reversal for excitation intensities above a certain threshold, can be achieved ${ }^{8}$. However, these effects were thought to occur only immediately beneath the surface of the material, that is, over the micrometre extinction depth of the resonantly driven phonon, which was probed by time-resolved second-harmonic generation. Here, by using a bulk-sensitive Raman scattering probe, we show that both reduction and reversal of the ferroelectric polarization occur over the entire $50 \mu \mathrm{m}$ thickness of the crystal, far beyond the excitation region.
The physical situation leading to nonlocal manipulation of the ferroelectric polarization is sketched in Fig. 1a and $1 \mathrm{~b}$. We only consider the $c$-axis-polarized $18.3 \mathrm{THz} \mathrm{A}_{1}$ mode $\left(Q_{\mathrm{IR}}\right)$, which is driven resonantly by the pump pulse, and the $7.3 \mathrm{THz} \mathrm{A}_{1}$ 'soft mode' ${ }^{16}\left(Q_{\mathrm{P}}\right)$, which is excited indirectly by anharmonic coupling to the driven mode. Note that there also exist two additional $A_{1}$ modes near 8 and $10 \mathrm{THz}$. However, their effective charges are respectively a factor of 30 and 8 smaller than those of $Q_{P}$ and hence they make negligible contributions to the time-dependent response of the material at terahertz frequencies.

The nonlinear response of the $\mathrm{LiNbO}_{3}$ during and after excitation is described by the following potential ${ }^{17,18}$ :

$$
\begin{aligned}
V\left(Q_{\mathrm{IR}}, Q_{\mathrm{P}}\right)= & \frac{1}{4}\left(-\omega_{\mathrm{P}}^{2} Q_{\mathrm{P}}^{2}+\frac{\kappa_{\mathrm{P}}}{2} Q_{\mathrm{P}}^{4}\right)+\frac{1}{2}\left(\omega_{\mathrm{IR}}^{2} Q_{\mathrm{IR}}^{2}+\frac{\kappa_{\mathrm{IR}}}{2} Q_{\mathrm{IR}}^{4}\right) \\
& +V_{\mathrm{c}}\left(Q_{\mathrm{IR}}, Q_{\mathrm{P}}\right)+V_{E}\left(Q_{\mathrm{IR}}, Q_{\mathrm{P}}\right)
\end{aligned}
$$

Here, ferroelectricity is modelled by a double-well potential along the soft mode coordinate $Q_{\mathrm{P}}$ with quartic nonlinear coefficient $\kappa_{\mathrm{P}}$ and minima located at $Q_{\mathrm{P}}= \pm \frac{P_{0}}{Z^{*}}$, where $P_{0}$ is the magnitude of the equilibrium ferroelectric polarization, and $Z_{\mathrm{P}}^{*}$ and $\omega_{\mathrm{p}}$ are, respectively, the effective charge and transverse optical frequency of the soft mode. The potential along $Q_{\mathrm{IR}}$ is accounted for in the form of a single parabolic well and additional quartic anharmonicity with coefficient $\kappa_{\mathrm{IR}}$, which is important to account for nonlinearities of its excitation process. The anharmonic potential term $V_{\mathrm{c}}\left(Q_{\mathrm{IR}}, Q_{\mathrm{P}}\right)=\alpha Q_{\mathrm{P}}^{2} Q_{\mathrm{IR}}^{2}+\beta Q_{\mathrm{P}} Q_{\mathrm{IR}}^{3}+\chi Q_{\mathrm{P}}^{3} Q_{\mathrm{IR}}$ describes the coupling between $Q_{\mathrm{IR}}$ and $Q_{\mathrm{P}}$, but also accounts for additional symmetry-odd terms in the $Q_{\text {IR }}$ potential that lead to higher-order phonon harmonics documented in ref. ${ }^{19}$. Finally, the term $V_{E}\left(Q_{\mathrm{IR}}, Q_{\mathrm{P}}\right)=E\left(Z_{\mathrm{P}}^{*} Q_{\mathrm{P}}+Z_{\mathrm{IR}}^{*} Q_{\mathrm{IR}}\right)$ accounts for the coupling of the modes to the excitation field $E$, where $Z_{\mathrm{IR}}^{*}$ is the effective charge of $Q_{\mathrm{IR}}$. Once the charged modes are set in motion, a time-dependent polarization $P=\epsilon_{0} \epsilon_{\infty} E+Z_{\mathrm{IR}}^{*} Q_{\mathrm{IR}}+Z_{\mathrm{P}}^{*} Q_{\mathrm{P}}$ is created, where $\varepsilon_{0}$ is the vacuum permittivity and $\varepsilon_{\infty}$ is the high-frequency dielectric constant.

Using the model above, the response of $\mathrm{LiNbO}_{3}$ to an incident mid-infrared pulse of duration $200 \mathrm{fs}$ and central frequency $20 \mathrm{THz}$

'Max Planck Institute for the Structure and Dynamics of Matter, Hamburg, Germany. ${ }^{2}$ Department of Physics, Clarendon Laboratory, University of Oxford,

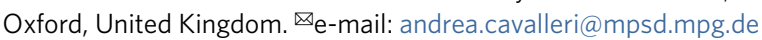


a

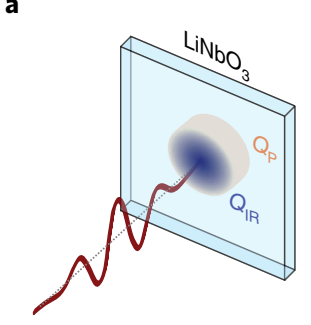

b

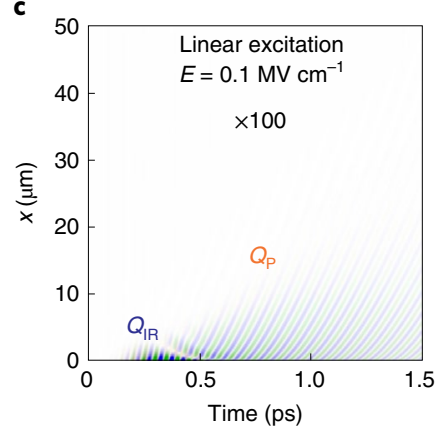

C

\begin{tabular}{|c|c|c|c|c|c|}
\hline \multirow{2}{*}{$Q_{\mathrm{IR}}$ (a.u.) } & & & & & \\
\hline & -1.0 & -0.5 & 0 & 0.5 & 1.0 \\
\hline \multirow[t]{2}{*}{$Q_{p}$ (a.u.) } & & & & & \\
\hline & -1.0 & -0.5 & 0 & 0.5 & 1.0 \\
\hline
\end{tabular}

Fig. 1 | Nonlocal nonlinear phononics. a, An evanescent excitation pulse drives a mode $Q_{\mathbb{R}}$ beneath the surface, which launches a propagating polariton mode $Q_{p}$ via anharmonic phonon coupling. b, Dispersion relation for $\mathrm{LiNbO}_{3}$ along the crystallographic $c$ axis. The parameters used to generate the curves were taken from ref. ${ }^{26}$. c, Simulations of the mode amplitudes $Q_{\mathbb{R}}$ and $Q_{p}$ upon excitation with a $200 \mathrm{fs}$ pulse tuned slightly above $\omega_{\mathbb{R}}$ at $20 \mathrm{THz}$ for an incident field strength of $E=0.1 \mathrm{MV} \mathrm{cm}^{-1}$. The vertical axis corresponds to the depth in the $\mathrm{LiNbO}_{3}$ sample. In addition to the evanescent excitation of $Q_{\mathbb{R}}$ at frequencies within the Reststrahlen band, the frequency components of the pulse just below the transverse optical phonon frequency $\omega_{\mathbb{R}}$ also excite a polariton of $Q_{\mathbb{R}}$ near $16 \mathrm{THz}$, which propagates deeper into the material and itself forces $Q_{P}$ to oscillate weakly at the same frequency. However, anharmonic phonon coupling is negligible and no frequency components outside the bandwidth of the excitation field are generated. $\mathbf{d}$, The same as $\mathbf{c}$, except with an incident field strength of $20 \mathrm{MV} \mathrm{cm}^{-1}$. Here, the nonlinear force on $Q_{p}$ becomes appreciable, resulting in the launching of a low-frequency polarization wave, which propagates throughout the medium. a.u., arbitrary units.

is readily simulated. Figure $1 \mathrm{c}$ shows the time- and space-dependent mode amplitudes $Q_{\mathrm{P}}$ and $Q_{\mathrm{IR}}$, simulated using finite-difference time-domain methods for excitation with a $100 \mathrm{kV} \mathrm{cm}^{-1}$ field. The frequency components of $Q_{\mathrm{IR}}$ excited at the transverse optical phonon frequency $\omega_{\mathrm{IR}}$ and within the reststrahlen band have the largest amplitudes and penetrate the material evanescently to depths on the order of a few micrometres. The frequency components of $Q_{I R}$ that are excited immediately below $\omega_{\mathrm{IR}}$ correspond to a polariton that propagates throughout the material. This polariton, which contains frequencies in the $16 \mathrm{THz}$ range, also off-resonantly excites $Q_{\mathrm{p}}$ causing it to weakly oscillate at the same frequency. Most notable is that all features shown in this panel are the result of a linear excitation in which anharmonic phonon coupling via the potential term $V_{\mathrm{c}}\left(Q_{\mathrm{IR}}, Q_{\mathrm{P}}\right)$ is negligible.

Figure 1d shows the calculated $Q_{\mathrm{IR}}$ and $Q_{\mathrm{P}}$ in the case of an excitation with a higher field strength of $20 \mathrm{MV} \mathrm{cm}^{-1}$. The features from the linear-excitation regime are still present; however, the nonlinear force on $Q_{\mathrm{P}}$ arising from $\alpha Q_{\mathrm{IR}}{ }^{2} Q_{\mathrm{P}}{ }^{2}$ in the $V_{\mathrm{c}}\left(Q_{\mathrm{IR}}, Q_{\mathrm{P}}\right)$ term is

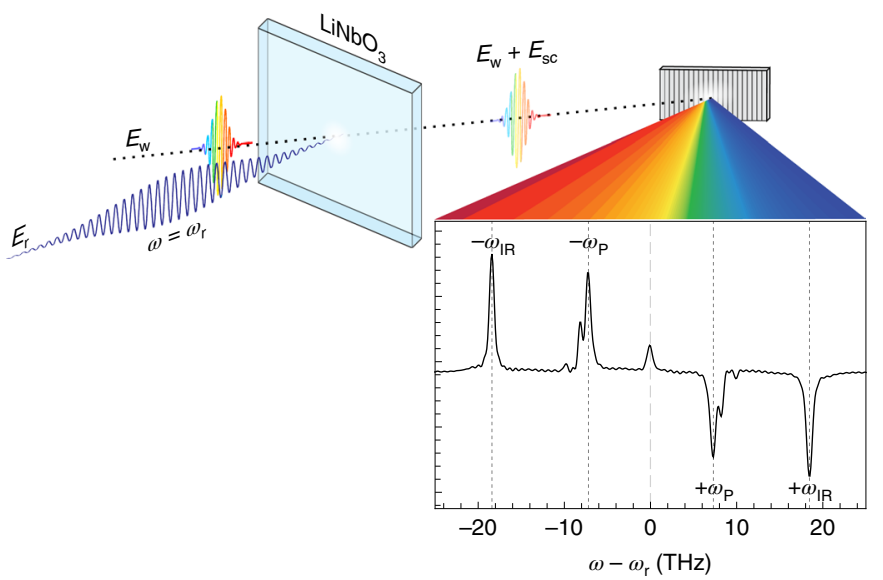

Fig. 2 | Femtosecond stimulated Raman scattering. A narrowband pulse $E_{\mathrm{r}}$ and a white-light continuum field $E_{\mathrm{w}}$ excite a coherent vibration with frequency $\omega_{\mathrm{Q}}$ with a force proportional to $E_{\mathrm{r}} E_{\mathrm{w}}$. The stimulated mode mixes with $E_{\mathrm{r}}$ via the Raman tensor element, resulting in the emission of sidebands at frequencies $\omega_{\mathrm{r}} \pm \omega_{\mathrm{Q}}$ that propagate in the same direction as $E_{\mathrm{w}}$. When sent to a spectrometer, the sideband fields and $E_{\mathrm{w}}$ interfere, resulting in the amplification of $E_{\mathrm{w}}$ at frequency $\omega_{\mathrm{r}}-\omega_{\mathrm{Q}}$ and depletion at $\omega_{\mathrm{r}}+\omega_{\mathrm{Q}}$. Shown is a typical FSRS spectrum of the $\mathrm{A}_{1}$ modes in $\mathrm{LiNbO}_{3}$.

now considerable. This anharmonic coupling term symmetrically displaces both wells of the potential towards $Q_{P}=0$. The rectified component of this force excites $Q_{\mathrm{P}}$ oscillations over a range of frequencies, which spans nearly the entire polariton branch. This results in a broadband, low-frequency $(f \approx 3 \mathrm{THz})$ polarization wave, which propagates throughout the crystal as shown in Fig. 1d. In real ferroelectric crystals, there exists also a contribution to the potential from uncompensated charges, which becomes relevant when $Q_{\mathrm{P}}$ is excited near the regime of polarization reversal ${ }^{17}$. However, for the perturbative regime considered so far, we neglect this effect.

The effects discussed above were probed through measuring the response of all the $A_{1}$ modes using femtosecond stimulated Raman spectroscopy (FSRS) ${ }^{20-22}$. In these FSRS experiments, two incident probe pulses were superimposed on the material. The first pulse, with electric field $E_{\mathrm{w}}$, was a white-light continuum pulse with a spectral bandwidth spanning tens of terahertz and temporal full-width at half-maximum (FWHM) of $\lesssim 100$ fs. The second pulse, with electric field $E_{\mathrm{r}}$, was centred at a wavelength of $400 \mathrm{~nm}$ and had a narrow spectral bandwidth less than $1 \mathrm{THz}$ and corresponding time duration of 3 ps. A sketch of the experimental set-up is shown in Fig. 2. The theory for FSRS has been discussed elsewhere ${ }^{23,24}$ and can be summarized as follows. When the two pulses impinge upon a given material, a force $F=R^{\star} E_{\mathrm{r}} E_{\mathrm{w}}$ is imparted onto a Raman-active mode $\mathrm{Q}$, which then oscillates at its eigenfrequency $\omega_{\mathrm{Q}}$. The oscillations at $\omega_{\mathrm{Q}}$ modulate the refractive index of the material, causing the narrowband pulse field $E_{\mathrm{r}}$ to develop Raman sidebands at $\omega_{\mathrm{r}} \pm \omega_{\mathrm{Q}}$. These sidebands are emitted in the direction of the white-light field $E_{\mathrm{w}}$, which after being sent through a spectrometer, interfere on a detector to yield heterodyned peaks that are positive at $\omega_{\mathrm{r}}-\omega_{\mathrm{Q}}$ and negative at $\omega_{\mathrm{r}}+\omega_{\mathrm{Q}}$. FSRS is especially fruitful because it allows for the detection of all Raman modes in a single probing event as well as ultrafast changes of the Raman coefficient $R^{\star}$.

In our experiments, FSRS spectra were recorded at each pumpprobe delay $\tau$ between the FSRS pulse pair and the mid-infrared pulse, as depicted in Fig. 3a (see Supplementary Section 4 for details). Figure $3 \mathrm{~b}$ shows the evolution of the experimental FSRS spectra with the time delay $\tau$ after illumination with a mid-infrared pulse of fluence $38 \mathrm{~mJ} \mathrm{~cm}^{-2}$ (field strength of $14 \mathrm{MV} \mathrm{cm}^{-1}$ ). Two 


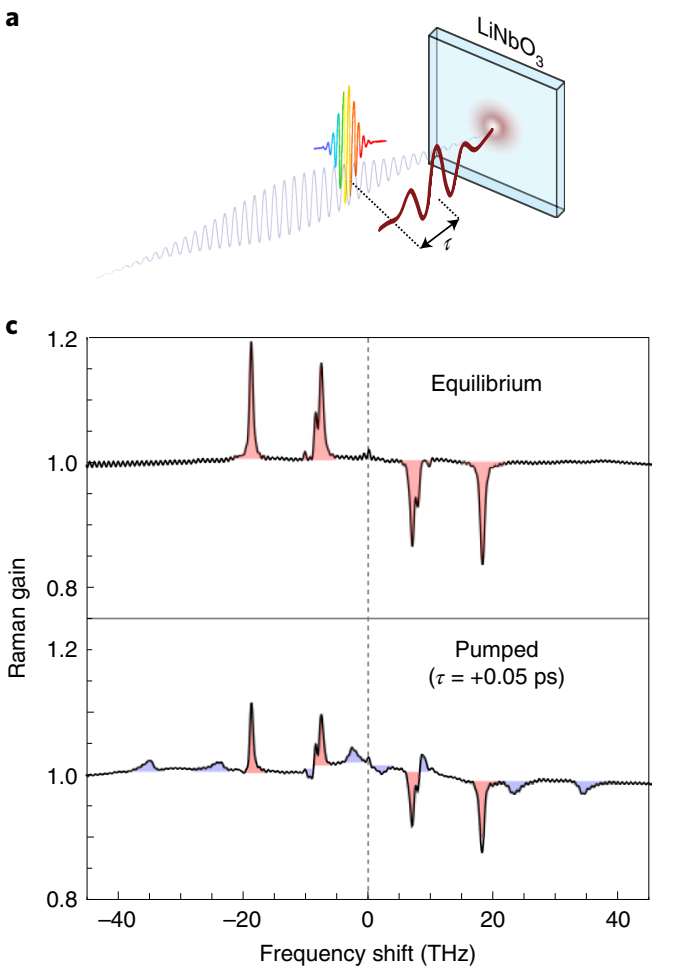

b

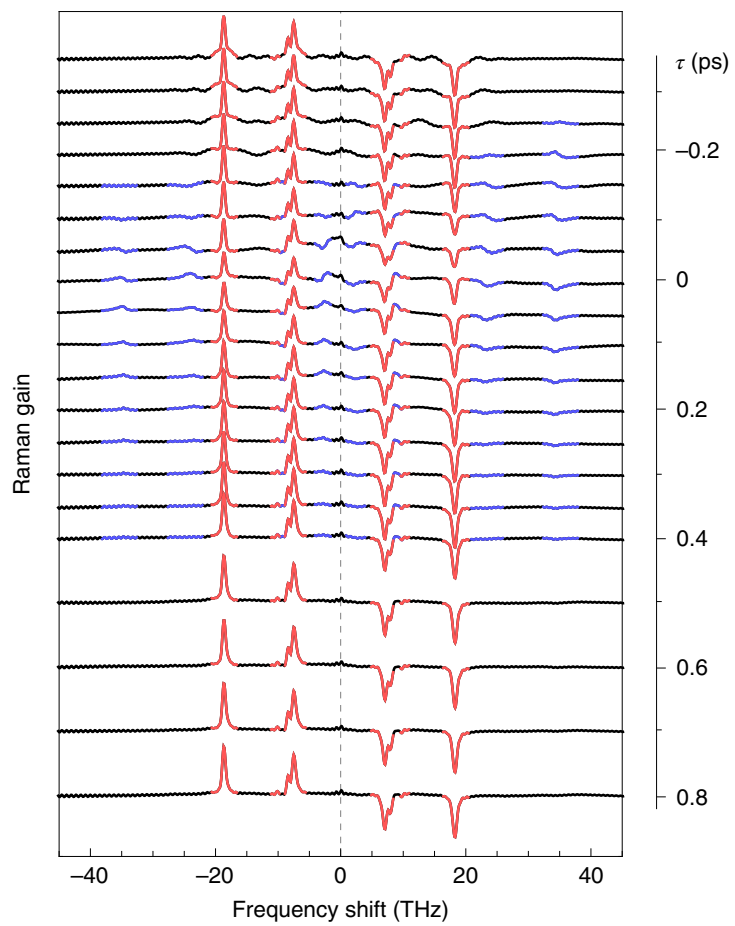

Fig. 3 | Time-resolved FSRS measurements of the $\mathrm{A}_{1}$ modes in $\mathrm{LiNbO}_{3}$. $\mathbf{a}$, Experimental geometry. Time-resolved changes to the FSRS spectra were recorded by varying the time delay $\tau$ between the FSRS pulse pair and the mid-infrared pump pulse. $\mathbf{b}$, FSRS spectra as a function of $\tau$ for a mid-infrared excitation fluence of $38 \mathrm{~mJ} \mathrm{~cm}^{-2}$ (14 $\mathrm{MV} \mathrm{cm}^{-1}$ field strength). The two prominent features that result from mid-infrared excitation are a reduction in the amplitudes of the $A_{1}$ modes (highlighted in red) and the emergence of new peaks (highlighted in blue). c, Equilibrium FSRS spectrum and pumped spectrum from $\mathbf{b}$ for $\tau=0.05 \mathrm{ps}$. As discussed in the text, the features in the pumped spectrum can be explained under the assumption that the Raman tensors for the $A_{1}$ modes change dynamically.
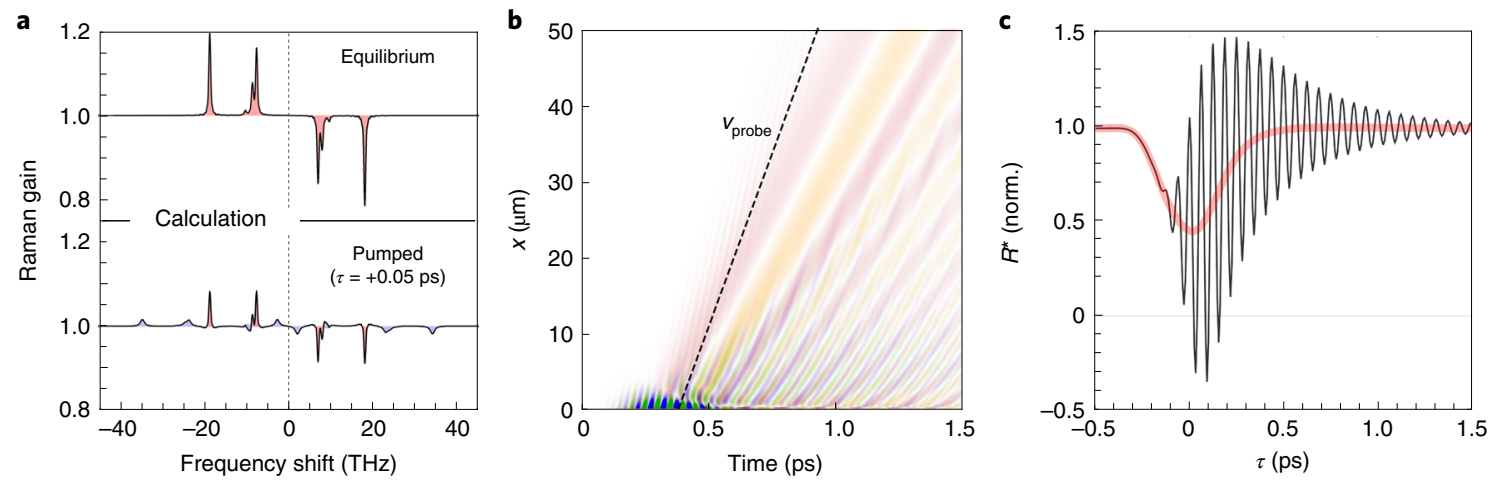

Fig. 4 | Calculations of pumped FSRS spectra in $\mathrm{LiNbO}_{3}$. $\mathbf{a}$, Equilibrium and pumped FSRS spectra for $\tau=0.05$ ps. The calculations were produced using a time-domain model for the FSRS process that is detailed in Supplementary Section 8. b. Group velocity of the FSRS probe projected onto the spacetime maps of $Q_{\mathbb{R}}$ and $Q_{p}$ from Fig. 1d. Over the course of the $50 \mu \mathrm{m}$ sample depth, the probe velocity $\left(v_{\text {probe }}\right)$ is approximately matched with the phase velocity of the soft-mode polariton. Here, the effective velocity of the probe was calculated given the group refractive index of $\mathrm{LiNbO}_{3}$ at $400 \mathrm{~nm}$ as well as the angle between the mid-infrared pump and white-light continuum pulse. c, Black, the dynamic Raman tensor used to produce the pumped calculations displayed in $\mathbf{a}$. Red, the transient reduction of the Raman tensor seen in the rest frame of the probe after accounting for the effects of velocity mismatch throughout the $50 \mu \mathrm{m}$ crystal thickness. The component that oscillates at $16 \mathrm{THz}$ imparts sidebands to the frequencies of the equilibrium modes, giving rise to the blue-highlighted peaks in $\mathbf{a}$.

notable changes to the spectra were observed and are highlighted in Fig. 3c, which displays the pumped spectrum for $\tau=0.05 \mathrm{ps}$ together with the equilibrium response. The transient spectra show (1) a reduction in the peak amplitudes of all the equilibrium Raman peaks (highlighted in red) and (2) the appearance of several new peaks (indicated in blue).
The most important observation is the reduction in the Raman peak amplitudes of $Q_{\mathrm{IR}}$ and $Q_{\mathrm{P}}$. This effect is modelled by assuming that the equilibrium components of both $R_{\mathrm{IR}}^{*}$ and $R_{\mathrm{P}}^{*}$ become transiently reduced upon excitation with the mid-infrared pulse (Fig. 4a). Because the peak amplitudes observed in FSRS scale exponentially with the sample thickness ${ }^{23,24}$, we conclude that the 
a

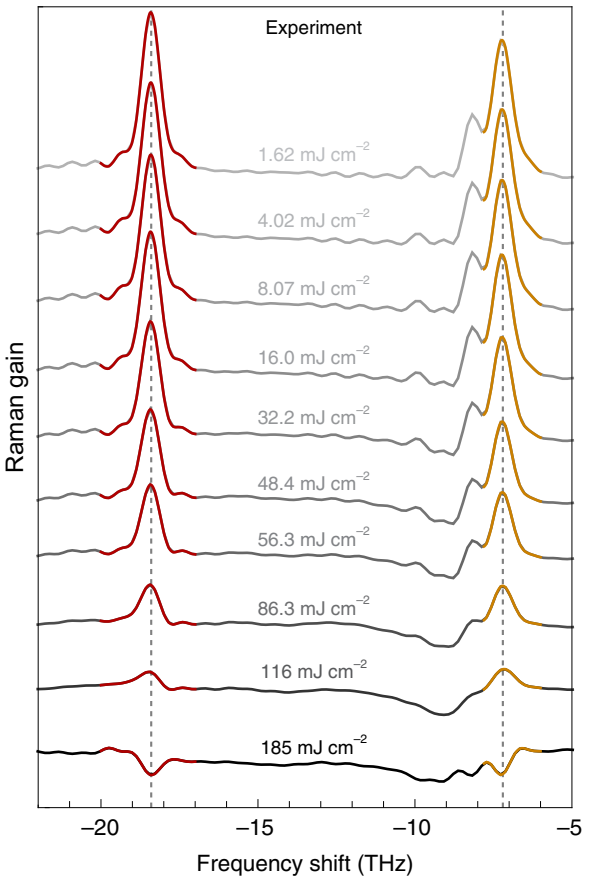

b
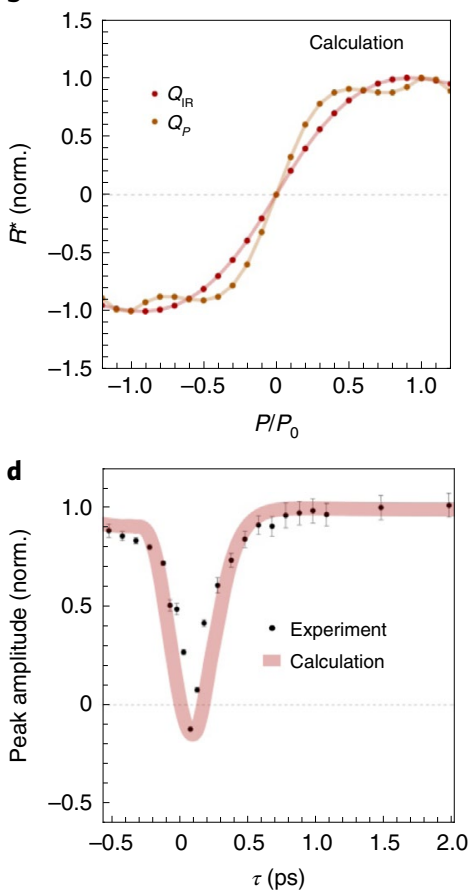

c
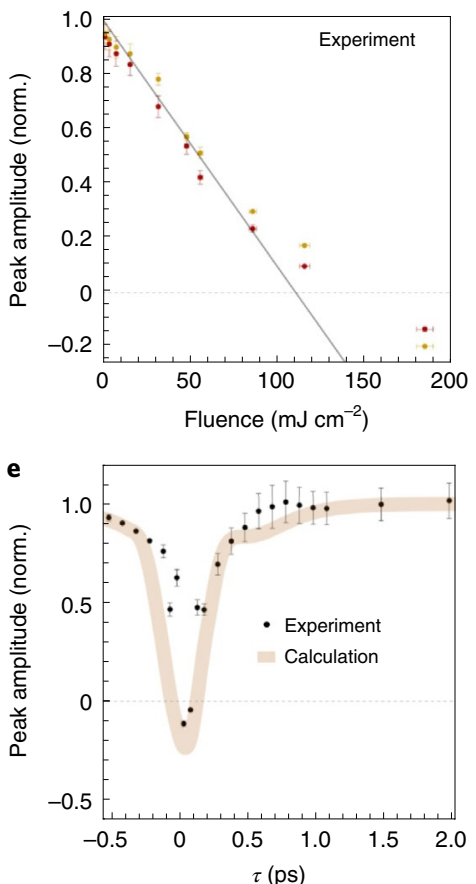

Fig. 5 | Reversal of the ferroelectric polarization in the bulk. a, Fluence dependence of the $A_{1}$ modes for $\tau=0.08$ ps. A vertical offset was applied to each spectrum for clarity. The FSRS peaks reverse sign at the highest fluence of $185 \mathrm{~mJ} \mathrm{~cm}^{-2}$ ( $30 \mathrm{MV} \mathrm{cm}^{-1}$ field strength). $\mathbf{b}$, First-principles calculations of the normalized Raman tensors for $Q_{\mathbb{R}}$ and $Q_{P}$ as a function of the ferroelectric polarization $P / P_{0}$, where $P_{0}$ is the value of the static polarization at room temperature and equilibrium. c, Amplitudes of the Raman peaks associated with $Q_{\mathbb{R}}$ and $Q_{p}$. Horizontal error bars indicate the systematic uncertainty in the fluence measurements. At fluences above approximately $60 \mathrm{~mJ} \mathrm{~cm}^{-2}\left(17 \mathrm{MV} \mathrm{cm}^{-1}\right.$ field strength), the reductions of the peak amplitudes begin to exhibit a saturation-like behaviour, possibly indicating a renormalization of the frequency of the driven mode due to a $Q_{\mathbb{R}}{ }^{4}$ term in the $c r y s t a l$ potential. $\mathbf{d}, \mathbf{e}$, The peak amplitudes as a function of $\tau$ at the highest excitation fluence of $185 \mathrm{~mJ} \mathrm{~cm}^{-2}$ for $Q_{\mathbb{I R}}(\mathbf{d})$ and $Q_{p}(\mathbf{e})$. The calculations were performed in the same manner as those of Fig. 4, except that here it is assumed that a transient sign reversal of the Raman tensor propagates throughout the medium, rather than a simple reduction. The results of $\mathbf{b}$ were used to model the corresponding Raman tensors for $Q_{\mathbb{R}}$ and $Q_{p}$. The peak amplitudes shown in c-e were extracted via Lorentzian fits to the FSRS spectra. Vertical error bars indicate the uncertainty of the extracted amplitudes as determined from the fit residuals.

optical excitation must be reducing the ferroelectric polarization, and thus $R_{\mathrm{IR}}^{*}$ and $R_{\mathrm{P}}^{*}\left(\right.$ ref. $\left.{ }^{25}\right)$, throughout the entire crystal $(50 \mu \mathrm{m}$ thick). Indeed, if these Raman coefficients were only modified within the few-micrometre penetration depth of the pump, the pump-induced changes to the FSRS spectra would not be observable in our experiment (Supplementary Section 2).

The observed appearance of new peaks in Fig. $3 \mathrm{c}$ is well explained by the direct modulation of the Raman tensors $R_{\mathrm{IR}}^{*}$ and $R_{\mathrm{P}}^{*}$ at the $16 \mathrm{THz}$ polariton frequency (Fig. 1c). Second-order Raman scattering is evidenced here by satellite peaks appearing at $\omega_{\mathrm{IR}} \pm 16 \mathrm{THz}$ and $\omega_{\mathrm{P}} \pm 16 \mathrm{THz}$.

The calculated spectra shown in Fig. 4a were obtained by assuming that the Raman tensors reduced over a timescale similar to those of the transient polarization reductions observed in ref. ${ }^{8}$. Our model assumed that these reductions of the Raman tensor propagated throughout the crystal at the phase velocity of the soft-mode polariton. To account for the propagation mismatch with the probe, the velocity line from Fig. $4 \mathrm{~b}$ was projected onto the spacetime map for the Raman tensor, and an integral along the velocity line was carried out for each time step. The result is highlighted in red in Fig. 4c. A damped sinusoid with a frequency of $16 \mathrm{THz}$ was then added to the result to account for the phase-matched detection of the $16 \mathrm{THz}$ modulation of the Raman tensor. The curve shown in black in Fig. $4 c$ models the effective $R^{\star}(t)$ seen by the probe and was used to produce the spectra shown in Fig. 4a. Complete details regarding the calculations are provided in Supplementary Section 8.
Figure $5 \mathrm{a}$ reports the experimentally determined fluence dependence of the peak reductions for a fixed $\tau=0.08 \mathrm{ps}$. As the mid-infrared excitation fluence was increased, the FSRS peaks decreased monotonically in amplitude and then reversed sign at the maximum fluence of $185 \mathrm{~mJ} \mathrm{~cm}^{-2}$ (field strength of $30 \mathrm{MV} \mathrm{cm}^{-1}$ ) used in the experiment. Note that the ability to detect a sign change of $R^{\star}$ is unique to the FSRS probe (discussed further in Supplementary Section 1). The results of our calculations, shown in Fig. 5b, confirm that if the ferroelectric polarization is reversed, the signs of both Raman tensors $R_{\mathrm{IR}}^{*}$ and $R_{\mathrm{P}}^{*}$ also reverse. Taken together with the experimental data, this theoretical consideration evidences a transient reversal of the ferroelectric polarization in the bulk crystal.

The amplitudes of the FSRS peaks corresponding to $Q_{I R}$ and $Q_{P}$ as a function of mid-infrared pump fluence are shown in Fig. $5 \mathrm{c}$. Note that for fluences above approximately $60 \mathrm{~mJ} \mathrm{~cm}^{-2}$ the peak amplitudes are no longer linear and tend to be already saturated before they reverse sign. One effect that would produce one such saturation would be a softening of the frequency of the driven mode $Q_{\mathrm{IR}}$ due to the fourth-order term $Q_{\mathrm{IR}}^{4}$ found in the potential of equation (1), an effect that reduces its response to the driving electric field. The evolution of the peak amplitudes with $\tau$ at maximum excitation fluence is presented in Fig. 5 d,e along with calculated values. Importantly, the calculations were performed in the same manner as were those shown in Fig. 4, but with the exception that here the Raman tensors of both modes are assumed to exhibit a transient sign change and subsequent recovery to their equilibrium values. 
While the change in sign of all of the Raman tensor elements indicates a reversal of the ferroelectric polarization, this regime cannot be described by the theory of propagating polaritons alone. What can be quantitatively predicted is that if a ferroelectric domain is reversed beneath the surface, as discussed in ref. ${ }^{8}$, a domain wall will propagate into the bulk while emitting coherent electromagnetic radiation. Qualitatively, we expect that this effect should cooperatively overcome the local coercive field as the ferroelectric domain-wall polarization propagates throughout the bulk at near-luminal speeds. Such a highly nonlinear process merits a more comprehensive theory and will be a subject of future studies.

\section{Online content}

Any methods, additional references, Nature Research reporting summaries, source data, extended data, supplementary information, acknowledgements, peer review information; details of author contributions and competing interests; and statements of data and code availability are available at https:/doi.org/10.1038/ s41567-022-01512-3.

Received: 5 May 2021; Accepted: 14 January 2022; Published online: 7 March 2022

\section{References}

1. Först, M. et al. Nonlinear phononics as an ultrafast route to lattice control. Nat. Phys. 7, 854-856 (2011).

2. Rini, M. et al. Control of the electronic phase of a manganite by mode-selective vibrational excitation. Nature 449, 72-74 (2007).

3. Nova, T. F. et al. An effective magnetic field from optically driven phonons. Nat. Phys. 13, 132-136 (2017).

4. Disa, A. S. et al. Polarizing an antiferromagnet by optical engineering of the crystal field. Nat. Phys. 16, 937-941 (2020).

5. Afanasiev, D. et al. Ultrafast control of magnetic interactions via light-driven phonons. Nat. Mater. 20, 607-611 (2021).

6. Fausti, D. et al. Light-induced superconductivity in a stripe-ordered cuprate Science 331, 189-191 (2011)

7. Mankowsky, R. et al. Nonlinear lattice dynamics as a basis for enhanced superconductivity in $\mathrm{YBa}_{2} \mathrm{Cu}_{3} \mathrm{O}_{6.5}$. Nature 516, 71-73 (2014).

8. Mankowsky, R., Von Hoegen, A., Först, M. \& Cavalleri, A. Ultrafast reversal of the ferroelectric polarization. Phys. Rev. Lett. 118, 197601 (2017).

9. Nova, T. F., Disa, A. S., Fechner, M. \& Cavalleri, A. Metastable ferroelectricity in optically strained $\mathrm{SrTiO}_{3}$. Science 364, 1075-1079 (2019).

10. Li, X. et al. Terahertz field-induced ferroelectricity in quantum paraelectric $\mathrm{SrTiO}_{3}$. Science 364, 1079-1082 (2019).

11. Adachi, S., Koehl, R. M. \& Nelson, K. A. Real-space and real-time imaging of polariton wavepackets. J. Lumin. 87, 840-843 (2000).
12. Stevens, T. E., Wahlstrand, J. K., Kuhl, J. \& Merlin, R. Cherenkov radiation at speeds below the light threshold: phonon-assisted phase matching. Science 291, 627-630 (2001).

13. Koehl, R. M. \& Nelson, K. A. Coherent optical control over collective vibrations traveling at lightlike speeds. J. Chem. Phys. 114, 1443-1446 (2001).

14. Feurer, T., Vaughan, J. C. \& Nelson, K. A. Spatiotemporal coherent control of lattice vibrational waves. Science 299, 374-377 (2003).

15. Fahy, S. \& Merlin, R. Reversal of ferroelectric domains by ultrashort optical pulses. Phys. Rev. Lett. 73, 1122-1125 (1994).

16. Servoin, J. L. \& Gervais, F. Soft vibrational mode in $\mathrm{LiNbO}_{3}$ and $\mathrm{LiTaO}_{3}$. Solid State Commun. 31, 387-391 (1979).

17. Abalmasov, V. A. Ultrafast reversal of the ferroelectric polarization by a midinfrared pulse. Phys. Rev. B 101, 014102 (2020).

18. Mertelj, T. \& Kabanov, V. V. Comment on 'Ultrafast reversal of the ferroelectric polarization'. Phys. Rev. Lett. 123, 129701 (2019).

19. Von Hoegen, A. Probing the interatomic potential of solids with strong-field nonlinear phononics. Nature 555, 79-82 (2018).

20. Dietze, D. R. \& Mathies, R. A. Femtosecond stimulated Raman spectroscopy. ChemPhysChem 17, 1224-1251 (2016).

21. Batignani, G. et al. Probing ultrafast photo-induced dynamics of the exchange energy in a Heisenberg antiferromagnet. Nat. Photon. 9, 506-510 (2015).

22. Kukura, P., McCamant, D. W., Yoon, S., Wandschneider, D. B. \& Mathies, R. A. Chemistry: structural observation of the primary isomerization in vision with femtosecond-stimulated Raman. Science 310, 1006-1009 (2005).

23. Lee, S. Y., Zhang, D., McCamant, D. W., Kukura, P. \& Mathies, R. A. Theory of femtosecond stimulated Raman spectroscopy. J. Chem. Phys. 121, 3632-3642 (2004).

24. Yoon, S. et al. Dependence of line shapes in femtosecond broadband stimulated Raman spectroscopy on pump-probe time delay. J. Chem. Phys. 122, 024505 (2005).

25. Ridah, A., Fontana, M. D. \& Bourson, P. Temperature dependence of the Raman modes in $\mathrm{LiNbO}_{3}$ and mechanism of the phase transition. Phys. Rev. B 56, 5967-5973 (1997).

26. Barker, A. S. \& Loudon, R. Dielectric properties and optical phonons in $\mathrm{LiNbO}_{3}$. Phys. Rev. 158, 433-445 (1967).

Publisher's note Springer Nature remains neutral with regard to jurisdictional claims in published maps and institutional affiliations.

Open Access This article is licensed under a Creative Commons Attribution 4.0 International License, which permits use, sharing, adaptation, distribution and reproduction in any medium or format, as long as you give appropriate credit to the original author(s) and the source, provide a link to the Creative Commons license, and indicate if changes were made. The images or other third party material in this article are included in the article's Creative Commons license unless indicated otherwise in a credit line to the material. If material is not included in the article's Creative Commons license and your intended use is not permitted by statutory regulation or exceeds the permitted use, you will need to obtain permission directly from the copyright holder. To view a copy of this license, visit http://creativecommons. org/licenses/by/4.0/.

(c) The Author(s) 2022 


\section{Data availability}

Source data are provided with this paper. All other source data related to the results discussed in this paper are available upon reasonable request.

\section{Acknowledgement}

We acknowledge support from the Deutsche Forschungsgemeinschaft via the Cluster of Excellence 'The Hamburg Centre for Ultrafast Imaging'.

\section{Author contributions}

The experiment was conceived by M.H., M. Först and A.C. M.H. designed and constructed the experimental set-up with input from M. Först. M.H. and E.R. carried out the experiments and collected data. Experimental data were processed by M.H. M. Fechner carried out all first-principles calculations and designed the finite-difference time-domain model of the polariton propagation. Simulations of the FSRS spectra were carried out by M.H. and M. Fechner. All the authors contributed to the discussion and interpretation of results. M.H., M. Först and A.C. wrote the manuscript with contributions from all authors.

\section{Funding}

Open access funding provided by Max Planck Society.

\section{Competing interests}

The authors declare no competing interests.

\section{Additional information}

Supplementary information The online version contains supplementary material available at https://doi.org/10.1038/s41567-022-01512-3.

Correspondence and requests for materials should be addressed to A. Cavalleri. Peer review information Nature Physics thanks Dominik Juraschek, Dmytro Afanasiev and the other, anonymous, reviewer(s) for their contribution to the peer review of this work.

Reprints and permissions information is available at www.nature.com/reprints. 